\title{
Keratoconus Features on Corneal Higher-Order Aberration Ablation Maps: Proof-of-Concept of a New Diagnostic Modality
}

This article was published in the following Dove Press journal: Clinical Ophthalmology

\author{
Avi Wallerstein (D) ${ }^{1,2}$ \\ Mathieu Gauvin (D) ${ }^{1,2}$ \\ Michael Mimouni ${ }^{3}$ \\ Louis Racine ${ }^{4}$ \\ Ali Salimi (iD $)^{1,2}$ \\ Mark Cohen ${ }^{2,5}$
}

'Department of Ophthalmology and Visual Sciences, McGill University, Montreal, QC, Canada; '2LASIK MD, Montreal, QC, Canada; ${ }^{3}$ Department of Ophthalmology and Vision Sciences, University of Toronto, Toronto, ON, Canada; ${ }^{4}$ Department of Ophthalmology, University of Montreal, Montreal, QC, Canada; ${ }^{5}$ Department of Surgery, University of Sherbrooke, Sherbrooke, QC, Canada
Correspondence: Avi Wallerstein 1250 Rene-Levesque Blvd W, MD Level, Montreal, QC, H3B 4W8, Canada Tel + I 514-908-9888, Ext. 2273

Email awallerstein@lasikmd.com
Purpose: To assess the potential application of corneal higher-order aberration (HOA) excimer ablation map imaging in identifying reproducible keratoconus (KC) features and to explore if newly derived map metrics correlate to Pentacam $\mathrm{KC}$ indices.

Methods: Case series of 12 eyes with $\mathrm{KC} \geq$ grade 2 . Topolyzer Vario corneal imaging with its resultant HOA ablation map islands were analyzed for their centroid, distance from center, angular position, orientation, sphericity, diameter, area, and maximal ablation depth. Correlations to Pentacam indices were studied.

Results: All eyes showed recurrent features with an arrangement of two elliptical paracentral ablation islands, one deep inferotemporal and one shallow superonasal, in direct mirror-like opposition to each other. These were always accompanied by superior peripheral ablation crescents. The two paracentral islands had highly reproducible distance from center $(1.2 \pm 0.1 \mathrm{~mm}$ and $1.3 \pm 0.2 \mathrm{~mm})$ and angular positions $\left(246.8 \pm 15.9^{\circ}\right.$ and $\left.76.7 \pm 7.7^{\circ}\right)$, with greater variation in ablation depth $(68.3 \pm 33.2 \mu \mathrm{m}$ and $17.6 \pm 12.1 \mu \mathrm{m})$. Distance from center of the peripheral superior crescents was highly reproducible $(3.3 \pm 0.1 \mathrm{~mm})$, with a larger range of depth $(74.5 \pm 37.2 \mu \mathrm{m})$. The deep paracentral inferotemporal island "hot spot" was coincident with the topographical apical cone. Strong correlations were found between the depth of the inferotemporal island and Pentacam indices of posterior radius curvature (PRC: $R=-0.74$ ) and Belin/Ambrosio enhanced ectasia total deviation (BAD-D: $R=0.71)$

Conclusion: The corneal HOA ablation map revealed a recurring, distinct, easily recognizable pattern in KC eyes. There was a strong correlation between the depth of novel HOA ablation map metrics and validated Pentacam KC indices. Novel information can be extracted from the corneal HOA ablation map giving it the potential to be a new modality to diagnose and grade $\mathrm{KC}$.

Keywords: topography, Contoura, keratoconus, ectasia, diagnosis, higher-order aberration

\section{Introduction}

Keratoconus $(\mathrm{KC})$ is a corneal ectatic condition with progressive corneal thinning and cone protrusion leading to irregular astigmatism that impairs vision. ${ }^{1}$ The reported prevalence of $\mathrm{KC}$ ranges between $0.0003 \%$ and $2.3 \%$ depending on the ethnicity, geographic location, and studied criteria for diagnosis. ${ }^{2-4}$ Diagnosing moderate or advanced $\mathrm{KC}$ using clinical findings coupled with topographic, topometric, tomographic, and biomechanical indices is straight forward. ${ }^{5,6}$ The challenge remains in detecting the disease in its earliest subclinical stage. Early 
detection of $\mathrm{KC}$ has gained importance to allow for corneal crosslinking, a procedure that delays or halts progression of disease. ${ }^{7}$ It is also imperative to identify and exclude $\mathrm{KC}$ and subclinical $\mathrm{KC}$ from corneal laser refractive surgery, since flap creation and tissue ablation further biomechanically weaken a predisposed cornea and can lead to postoperative ectasia. ${ }^{8-10}$

The accuracy of diagnostic identifiers for early $\mathrm{KC}$ screening has been a topic of various studies. The anterior and posterior surface elevations, the corneal thickness profile, the corneal epithelial patterns, wavefront aberration metrics, and corneal biomechanics have all been reported as indicators to diagnose KC. ${ }^{11-13}$ No single diagnostic parameter has been found to be sensitive enough to differentiate normals from early KC. Decision making has therefore been guided by combining several metrics as well as indices from different technologies, with the use of algorithmic models.

The following case series sets out to identify features of the corneal higher-order aberration (HOA) excimer ablation map in $\mathrm{KC}$ eyes in order to investigate this imaging modality's diagnostic feasibility. The imaging is derived from the Alcon platform for topography-guided excimer treatments that use the WaveLight ${ }^{\circledR}$ Topolyzer ${ }^{\mathrm{TM}}$ VARIO high-resolution Placido disc topographer to image the cornea. The topographic data is then used by the excimer laser Contoura treatment software to generate an ablation map that combines both lower-order aberration and HOA data into one image, providing ablation depth data. ${ }^{14}$ By inputting the sphere and cylinder treatment to zero in the Contoura planning software, one can separate out and exclusively see the anterior corneal HOA ablation map, which has clinical utility to graphically describe the exact location and depth of anterior corneal HOAs to be treated by the excimer laser. ${ }^{14-17}$ HOA ablation maps contain the information used by the excimer laser to ablate cornea and are therefore used for treatment purposes. HOA ablation map patterns and ablation depth have been previously reported in virgin corneas ${ }^{14}$ and highly aberrated corneas, ${ }^{18}$ but have not been used for diagnosis of corneal pathology or to characterize patients with KC.

This pilot study aims to assess the potential applications of HOA ablation maps in identifying and quantitively characterizing reproducible, original features in $\mathrm{KC}$ eyes, and to determine if correlations exist between these novel $\mathrm{KC}$ attributes and Pentacam indices used for $\mathrm{KC}$ diagnosis.

\section{Methods}

\section{Selection of Keratoconus Patients and Keratoconus Grading}

Patients diagnosed with keratoconus grade 2 and above at the time of their preoperative laser refractive surgery consultation were identified. Keratoconus diagnosis and grading was determined by a cornea specialist (LR) based on the modified Amsler-Krumeich criteria ${ }^{19-21}$ and the Belin ABCD Keratoconus Staging scale. ${ }^{22}$ We did not include early, subclinical, and grade $1 \mathrm{KC}$, to ensure that true $\mathrm{KC}$ eyes were being studied. Only eyes with definitive diagnostic KC topographies were included as a necessary scientific first step. In addition to a complete slit-lamp examination, corneas were only included if Pentacam tomography (Oculus, Lynwood, WA), Corvis biomechanical analysis (Oculus), and corneal epithelium thickness map imaging using an anterior segment optical coherence tomography (iVue, Optovue, Fremont, CA, USA) were available. The rationale for using multiple measures was to validate objective assessments that confirm the diagnosis of grade $2 \mathrm{KC}$ and above. This retrospective study was approved by the Ethics Review Board of the Canadian Ophthalmic Research Centre. All patients provided written consent for use of anonymized data for research. All experiments fulfilled the principles of the Declaration of Helsinki.

\section{Topography Acquisition}

As previously described, ${ }^{14,17,23,24}$ from 4 to 8 corneal topographies were acquired on undilated eyes with the WaveLight $^{\circledR}$ Topolyzer ${ }^{\mathrm{TM}}$ VARIO (Alcon). The criteria for image acceptance included appropriate recognition of the pupil and the mire edge by the software, mires with minimal breaks, the absence of significant missing data (shadow from the lids, lashes, nose, or dry tear film), and a percentage of data obtained (Analyzed Area, AA) greater than $90 \%$ in the $6.5 \mathrm{~mm}$ zone. Maps were compared to assess the reproducibility of the scans including keratometry, $\mathrm{Q}$ value, and the axis of astigmatism. Cases were then exported to the Contoura software to generate the HOA ablation profiles.

\section{Corneal HOA Ablation Map Image Production and Analysis}

Contoura HOA ablation map image was produced using a $6.5 \mathrm{~mm}$ optical zone as described previously. ${ }^{14,17,23}$ Briefly, the ablation map generated by the Contoura software 
includes both lower-order aberrations and HOAs. The isolated HOA ablation profile was obtained by entering a sphere and cylinder treatment of zero in the Contoura treatment planning software. The HOA ablation pattern was verified to be consistent with anterior elevation topography. The HOA ablation map scale is normalized (fixed setting) to its deepest ablation value, resulting in each map having the same range of colors, irrespective of the level of depth. Yellow/green colors always indicate the shallower ablation values for that specific map, while red/purple colors always indicate deeper ablation values. When the grid option is turned on, the HOA map fixed ring markings represent 2, 4,6 , and $8 \mathrm{~mm}$ diameter circles. We termed ablation islands inside the $3 \mathrm{~mm}$ diameter zone as paracentral and those outside the $3 \mathrm{~mm}$ diameter zone as peripheral.

HAO Ablation maps were imported in MATLAB R2020a (MathWorks, Natick, MA, USA) and quantitatively characterized with the Image Processing Toolbox ${ }^{\mathrm{TM}}$ (MATLAB). MATLAB ${ }^{\circledR}$ is a programming platform used to analyze data, perform statistical analyses, and to create algorithms. The MATLAB's Image Processing Toolbox provides standardized algorithms and tools for image visualization, segmentation, and analyses. Deep (red/purple on the color scale) and shallow (yellow/green on the color scale) ablation areas were identified and classified as distinct ablation islands. Up to 4 recurring ablation islands were identified on each HOA ablation map, and their respective centroid, distance from center, position on the map (between 0 and 360 degrees), orientation (between 0 and 180 degrees), sphericity, diameter (maximal length of the ablation island in $\mathrm{mm}$ ), area (in $\mathrm{mm}^{2}$ ) and maximal ablation depth (in $\mu \mathrm{m}$ ) were calculated (see methodological details in Figure $1 \mathrm{~A}-\mathrm{C}$ ). Ablation island centroids were calculated as the arithmetic mean of the coordinates of the pixels forming the ablation island in $\mathrm{x}$ and $\mathrm{y}$ dimensions. Each identified ablation island was also highlighted with a white outline and numbered \#1 to \#4.

\section{Data and Statistical Analysis}

Statistical analyses were conducted in MATLAB R2020a. Mean and standard deviation (SD) of each feature were calculated. The coefficient of variation (CV) was computed as the SD divided by the mean and multiplied by 100. The Pearson correlation coefficient (R-value) was used to assess the relationship between continuous variables. Statistical significance was set at $P<0.05$.

\section{Results}

There were 12 consecutive eyes of 10 patients diagnosed with $\mathrm{KC}$ grade 2 and above that met the inclusion criteria. The patients' average age was $37.8 \pm 11.2$ years (range: 27-61 years). Pentacam scans (4 Maps -

\section{Graphical Representation of HOA Ablation Map Measurement}

1 = Deep peripheral superior crescent
$2=$ Shallow paracentral superonasal island
$3=$ Deep paracentral inferaotemporal island
$4=$ Deep peripheral crescent

Figure I (A) Contoura HOA ablation map in a representative keratoconus eye. (B) Graphical representation of the distance from center and position features of an ablation island. The distance from center $(\rho)$ is defined as the radius (in $\mathrm{mm}$ ) between the origin of the HOA ablation map (X $=0$ and $Y=0$ coordinates) and the centroid of any ablation island. The centroid denoted by the white asterisk $\left(^{*}\right)$ is calculated as the arithmetic mean of the $X$ and $Y$ coordinates of all pixels forming the ablation island (in this example, all purple pixels). The orientation $(\theta)$ of the ablation island is defined as the angle (between 0 to $360^{\circ}$ ) formed between the centroid of the ablation island and the abscissa (dotted line). (C) Graphical representation of the orientation and diameter features of an ablation island. The orientation ( $\alpha$ ) of an ablation island is defined as the angle (reported between 0 to $180^{\circ}$ ) formed between the longest diagonal of the ablation island (termed major axis in geometry) and the dotted horizontal line passing by the centroid of the ablation island. The diameter of an ablation island was defined as the length of the major axis (d). In addition to the above-defined parameters, we calculated the area $\left(\mathrm{mm}^{2}\right)$ and sphericity of each ablation island. The area was calculated by multiplying the number of pixels by the area of one pixel. The sphericity of an ablation island was defined as $4 \Pi$ times area divided by the square of the perimeter. Sphericity is equal to I.0 for a perfect circle and it is smaller than I.0 for an ellipse or a crescent shape. 
Refractive view) revealed imaging consistent with $\mathrm{KC}$ (Figure 2). See Table 1 for individual Pentacam indices and group data. Corneal OCT epithelium mapping revealed abnormal epithelium profiles in 11 of 12 eyes (91.6\%), showing significant paracentral inferotemporal epithelium thinning, superoperipheral epithelium thickening, or a partial characteristic donut pattern (Figure 2). The Corvis Biomechanical Index (CBI) and Tomographic Biomechanical Index (TBI) were abnormal and equal to 1.0 in all eyes (Table 1).

\section{Individual HOA Ablation Maps, Corneal} Epithelium, and Pentacam Imaging

KC \#01

Figure 2 Individual HOA ablation maps, epithelium thickness profiles and Pentacam imaging data (4 Maps - Refractive view). 
Table I Description of Pentacam/Corvis Indices and Keratoconus Grade

\begin{tabular}{|c|c|c|c|c|c|c|c|c|c|c|c|}
\hline Case & Kmax & ARC & PRC & TP & ARTmax & PPI & BAD-D & CBI & TBI & A-K Grade & ABCD Grade \\
\hline KC \#0I & 55.4 & 6.6 & 4.9 & 415 & 12 & 2.5 & 9.6 & 1.0 & 1.0 & 2 & $\mathrm{~A} 2 \mathrm{~B} 4 \mathrm{C} 2 \mathrm{DI}$ \\
\hline KC \#02 & 61.0 & 6.1 & 4.4 & 428 & 93 & 2.7 & 12.9 & 1.0 & 1.0 & 2 & $\mathrm{~A} 4 \mathrm{~B} 4 \mathrm{C} 2 \mathrm{D} 2$ \\
\hline KC \#03 & 54.1 & 6.6 & 4.9 & 481 & $|4|$ & 2.4 & 7.6 & 1.0 & 1.0 & 2 & A2B4CIDI \\
\hline KC \#04 & 61.7 & 6.2 & 4.6 & 373 & 116 & 2.3 & 11.5 & 1.0 & 1.0 & 4 & $\mathrm{~A} 3 \mathrm{~B} 4 \mathrm{C} 3 \mathrm{D} 2$ \\
\hline KC \#05 & 58.7 & 6.1 & 4.3 & 446 & 109 & 2.9 & 14.6 & 1.0 & 1.0 & 2 & $\mathrm{~A} 4 \mathrm{~B} 4 \mathrm{C} 2 \mathrm{~B} 2$ \\
\hline KC \#06 & 74.1 & 5.7 & 4.1 & 451 & 94 & 2.8 & 14.8 & 1.0 & 1.0 & 4 & A4B4CID2 \\
\hline KC \#07 & 81.4 & 5.9 & 4.5 & 448 & 150 & 2.1 & 10.9 & 1.0 & 1.0 & 2 & $\mathrm{~A} 4 \mathrm{~B} 4 \mathrm{C} 2 \mathrm{D} 2$ \\
\hline KC \#08 & 50.5 & 7.1 & 5.5 & 511 & 283 & 1.4 & 2.8 & 1.0 & 1.0 & 2 & AIB2COD0 \\
\hline KC \#09 & 63.6 & 5.9 & 4.4 & 444 & 117 & 2.4 & 15.1 & 1.0 & 1.0 & 4 & $\mathrm{~A} 4 \mathrm{~B} 4 \mathrm{C} 2 \mathrm{D} 3$ \\
\hline KC \#10 & 56.0 & 6.9 & 4.3 & 443 & 100 & 2.8 & 12.6 & 1.0 & 1.0 & 2 & $\mathrm{~A} 2 \mathrm{~B} 4 \mathrm{C} 3 \mathrm{DI}$ \\
\hline KC \#II & 58.9 & 6.9 & 4.2 & 433 & 97 & 2.8 & 12.9 & 1.0 & 1.0 & 2 & A2B4C3DI \\
\hline KC \#12 & 63.2 & 6.0 & 4.2 & 485 & 137 & 2.5 & 11.9 & 1.0 & 1.0 & 3 & $\mathrm{~A} 4 \mathrm{~B} 4 \mathrm{CID} 2$ \\
\hline Average & $61.6 \pm 8.6$ & $6.3 \pm 0.5$ & $4.5 \pm 0.4$ & $447 \pm 35.4$ & $121 \pm 62.1$ & $2.5 \pm 0.4$ & $11.4 \pm 3.5$ & $1.0 \pm 0.0$ & $1.0 \pm 0.0$ & $2.6 \pm 0.9$ & A3B4C2D2 \\
\hline
\end{tabular}

Abbreviations: $\mathrm{Kmax}$, maximal keratometry; ARC, anterior radius of curvature; PRC, posterior radius of curvature; TP, thinnest point; ARTmax, maximum ambrosio relational thickness; PPI, Pentacam progression index; BAD-D, Belin/Ambrosio enhanced ectasia total Deviation; CBI, Corvis biomechanical index; TBI, tomographic biomechanical index; A-K Grade, keratoconus grade based on the modified Amsler-Krumeich criteria; $A B C D$ Grade, keratoconus grade based on the Pentacam Belin ABCD Keratoconus Staging.

\section{Characterization of 4 Ablation Islands on the Corneal HOA Ablation Map and Associated Reproducibility}

Individual KC HOA ablation maps are shown in Figure 3. Each map was found to have the following ablation islands: One (66.6\% of eyes) or two (33.3\% of eyes) red/ purple deep superior peripheral crescents (identified as \#1 and \#4) found in the superior quadrant in $94 \%$ of eyes and oriented nasally in $70 \%$ of eyes; a yellow/green shallow paracentral superonasal ablation island (identified as \#2), superonasal in $100 \%$ of eyes; and a deep red/purple paracentral inferotemporal ablation island (identified as \#3), located in the inferior quadrant in $100 \%$ of eyes, and temporal in $91.6 \%$ of eyes.

Both the yellow/green paracentral superonasal ablation islands and the red/purple paracentral inferotemporal ablation islands were elliptical in shape (Sphericity: $71.4 \pm 9.2 \%$ and $83.5 \pm 13.2 \%$; Table 2), of small diameter $(3.2 \pm 0.5 \mathrm{~mm}$ and $2.9 \pm 0.8 \mathrm{~mm})$, and of identical area $\left(3.6 \pm 0.9 \mathrm{~mm}^{2}\right.$ and $3.6 \pm 0.8 \mathrm{~mm}^{2}$ ), while the red/purple peripheral superior crescents were larger in area and longer in diameter, with much smaller sphericity indices $(29.3 \pm 12.1)$ due to their crescent-like shapes. The overall shapes are illustrated in Figure 4B, averaging all HOA ablation maps.

Distance from center, position, orientation, sphericity, diameter, area, and depth (methods described in Figure 1A-C) are reported for all 4 ablation islands in Figure 3. Group data reveals that the yellow/green paracentral superonasal ablation island (Figure 4A; green circles) and the red/purple paracentral inferotemporal ablation island (Figure 4A; red diamonds) were both highly reproducible in terms of distance from center $(1.3 \pm 0.2 \mathrm{~mm}$ and $1.2 \pm 0.1 \mathrm{~mm} ; \mathrm{CV}: 12.9 \%$ and $11.9 \%)$ and angular position $\left(76.7 \pm 7.7^{\circ}=\right.$ superonasal and $246.8 \pm 15.9^{\circ}$ $=$ inferotemporal; $\mathrm{CV}: 10.0 \%$ and $6.5 \%$ ), while their ablation depth varied more $(17.6 \pm 12.1 \mu \mathrm{m}$ and $68.3 \pm 33.2 \mu \mathrm{m}$; CV: $68.8 \%$ and $48.6 \%$ ). Similarly, the superior peripheral ablation crescents (Figure 4A, purple markers) were highly reproducible in terms of distance from center $(3.3 \pm 0.1 \mathrm{~mm} ; \mathrm{CV}: 2.4 \%)$, with a superonasal angular position $\left(77.9 \pm 27.1^{\circ} ; \mathrm{CV}: 34.8 \%\right)$, and a larger range of depth $(74.5 \pm 37.2 \mu \mathrm{m} ; \mathrm{CV}: 49.9 \%)$. All ablation island data and $\mathrm{CV}$ are reported in Table 2.

Strong correlations were found between the depth of the deepest peripheral crescent and the depth of the paracentral inferotemporal ablation island $(R=0.94 ; P<0.0001)$, between both the angular positions and the depths of the two paracentral ablation islands (angular position: $R=0.81 ; P=0.0014$; depth: $R=0.76 ; P=0.0044$ ). The angular position of the paracentral ellipses was also highly correlated to the angular position of the peripheral crescent $(R=0.68 ; P=0.0152)$.

\section{Correlations Between Ablation Island Features and Pentacam Indices}

Given that the ablation depth showed a larger range of values and a higher $\mathrm{CV}$ for all ablation islands, we examined if the ablation depth parameter correlated to $\mathrm{KC}$ grade. Pearson correlations between the ablation island depths and 8 different Pentacam indices (Kmax, ARC, PRC, TP, ARTmax, PPI, BAD-D, and ACA; Table 3) are reported. Strong correlations were found between maximal 
Quantitative Measures of Individual HOA Ablation Maps

\begin{tabular}{|c|c|c|c|c|c|}
\hline Island \# & 1 & 2 & 3 & 4 & \multirow{8}{*}{ KC\#1 } \\
\hline Distance & 3.4 & 1.4 & 1.2 & 3.2 & \\
\hline Position & 102.4 & 81.6 & 241.7 & 4.3 & \\
\hline Orientation & 10.3 & 172.8 & 172.8 & 95.3 & \\
\hline Sphericity & 33.1 & 72.1 & 96.1 & 45.9 & \\
\hline Diameter & 5.5 & 3.3 & 2.5 & 2.7 & \\
\hline Area & 4.6 & 3.8 & 3.9 & 1.4 & \\
\hline Depth & 39.7 & 3.7 & 32.2 & 30.3 & \\
\hline Island \# & 1 & 2 & 3 & 4 & \multirow[t]{8}{*}{ KC \#10 } \\
\hline Distance & 3.3 & 1.4 & 1.2 & N/A & \\
\hline Position & 79.7 & 83.8 & 257.5 & N/A & \\
\hline Orientation & 168.1 & 170.4 & 169.4 & N/A & \\
\hline Sphericity & 19.6 & 80.4 & 80.3 & N/A & \\
\hline Diameter & 9.5 & 2.8 & 3.2 & N/A & \\
\hline Area & 8.1 & 5.4 & 3.1 & N/A & \\
\hline Depth & 83.9 & 31.2 & 86.1 & N/A & \\
\hline Island \# & 1 & 2 & 3 & 4 & \multirow[t]{8}{*}{ 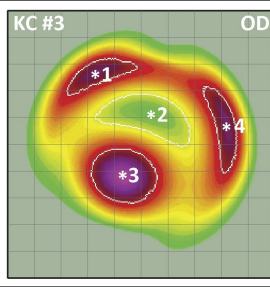 } \\
\hline Distance & 3.1 & 1.1 & 1.3 & 3.2 & \\
\hline Position & 120.8 & 75.5 & 239.3 & 59.4 & \\
\hline Orientation & 20.7 & 164.9 & 165.3 & 102.4 & \\
\hline Sphericity & 49.8 & 56.9 & 89.4 & 39.0 & \\
\hline Diameter & 2.8 & 3.7 & 2.5 & 3.4 & \\
\hline Area & 1.8 & 4.2 & 3.9 & 2.1 & \\
\hline Depth & 25.8 & 6.6 & 30.5 & 26.1 & \\
\hline Island \# & 1 & 2 & 3 & 4 & \multirow[t]{8}{*}{\begin{tabular}{|ll}
$K C$ & $O D$
\end{tabular}} \\
\hline Distance & 3.3 & 1.4 & 1.3 & N/A & \\
\hline Position & 58.9 & 60.5 & 234.9 & N/A & \\
\hline Orientation & 147.5 & 158.1 & 165.4 & N/A & \\
\hline Sphericity & 20.4 & 75.8 & 91.7 & N/A & \\
\hline Diameter & 9.3 & 3.2 & 2.5 & N/A & \\
\hline Area & 6.8 & 3.7 & 3.6 & N/A & \\
\hline Depth & 74.4 & 19.8 & 70.4 & N/A & \\
\hline Island \# & 1 & 2 & 3 & 4 & \multirow[t]{8}{*}{\begin{tabular}{|ll}
$K C H 7$ & $O D$
\end{tabular}} \\
\hline Distance & 3.4 & 1.6 & 1.0 & N/A & \\
\hline Position & 91.5 & 75.0 & 234.5 & N/A & \\
\hline Orientation & 179.1 & 163.2 & 161.8 & N/A & \\
\hline Sphericity & 30.5 & 70.1 & 90.3 & N/A & \\
\hline Diameter & 6.4 & 3.1 & 2.1 & N/A & \\
\hline Area & 5.1 & 3.0 & 2.1 & N/A & \\
\hline Depth & 134.4 & 14.3 & 112.6 & N/A & \\
\hline
\end{tabular}

\begin{tabular}{|c|c|c|c|c|c|c|}
\hline Island \# & 1 & 2 & 3 & 4 & \multirow{8}{*}{\multicolumn{2}{|c|}{$\begin{array}{ll}K C \# 8 & 0\end{array}$}} \\
\hline Distance & 3.3 & 1.1 & 1.4 & 2.9 & & \\
\hline Position & 147.5 & 108.4 & 265.2 & 36.1 & & \\
\hline Orientation & 56.0 & 162.9 & 166.6 & 141.2 & & \\
\hline Sphericity & 55.9 & 89.4 & 78.2 & 77.6 & & \\
\hline Diameter & 3.5 & 2.4 & 3.2 & 1.6 & & \\
\hline Area & 2.6 & 2.9 & 3.7 & 1.2 & & \\
\hline Depth & 12.2 & 3.4 & 11.6 & 10.1 & & \\
\hline Island \# & 1 & 2 & 3 & 4 & \multirow[t]{8}{*}{$\overline{K C} \# 2$} & os \\
\hline Distance & 3.3 & 1.1 & 1.2 & 3.3 & & \\
\hline Position & 93.7 & 98.1 & 297.4 & 210.3 & & \\
\hline Orientation & 7.9 & 15.7 & 17.7 & 121.3 & & \\
\hline Sphericity & 26.7 & 74.6 & 45.6 & 31.8 & & \\
\hline Diameter & 6.3 & 2.2 & 5.2 & 2.8 & & \\
\hline Area & 5.6 & 1.7 & 5.4 & 1.1 & & \\
\hline Depth & 112.4 & 36.8 & 111.5 & 93.0 & & \\
\hline Island \# & 1 & 2 & 3 & 4 & \multirow[t]{8}{*}{$\sqrt{\text { KC } \# 9}$} & os \\
\hline Distance & 3.3 & 1.4 & 1.2 & N/A & & \\
\hline Position & 105.8 & 104.4 & 270.0 & N/A & & \\
\hline Orientation & 177.3 & 10.4 & 11.1 & N/A & & \\
\hline Sphericity & 20.0 & 69.8 & 83.5 & N/A & & \\
\hline Diameter & 8.0 & 3.3 & 2.8 & N/A & & \\
\hline Area & 6.2 & 3.3 & 3.4 & N/A & & \\
\hline Depth & 100.1 & 26.3 & 76.8 & N/A & & \\
\hline Island \# & 1 & 2 & 3 & 4 & \multirow[t]{8}{*}{$\longdiv { \text { KC \#4 } }$} & OS \\
\hline Distance & 3.3 & 1.5 & 1.1 & N/A & & \\
\hline Position & 94.3 & 99.0 & 299.7 & N/A & & \\
\hline Orientation & 6.0 & 7.2 & 9.0 & N/A & & \\
\hline Sphericity & 30.9 & 63.7 & 83.5 & N/A & & \\
\hline Diameter & 5.2 & 3.9 & 2.6 & N/A & & \\
\hline Area & 3.7 & 4.2 & 3.1 & N/A & & \\
\hline Depth & 83.6 & 16.0 & 52.2 & N/A & & \\
\hline Island \# & 1 & 2 & 3 & 4 & \multirow[t]{8}{*}{ KC\#11 } & OS \\
\hline Distance & 3.3 & 1.3 & 1.3 & N/A & & \\
\hline Position & 103.2 & 91.8 & 280.3 & N/A & & \\
\hline Orientation & 8.8 & 2.7 & 5.6 & N/A & & \\
\hline Sphericity & 20.4 & 69.6 & 88.1 & N/A & & \\
\hline Diameter & 8.1 & 3.4 & 3.0 & N/A & & \\
\hline Area & 6.3 & 3.8 & 4.3 & N/A & & \\
\hline Depth & 100.0 & 34.6 & 99.9 & N/A & & \\
\hline
\end{tabular}

Figure 3 Individual HOA ablation maps. On each map, yellow/green colors indicate shallower ablation values and red/purple colors deeper ablation values. Each map is normalized to its deepest ablation value, so that all maps comprise the same range of colors. There were one or two red/purple peripheral ablation crescents (identified as Island \#I and \#4), a yellow/green paracentral superonasal ablation island (Island \#2), and a red/purple paracentral inferotemporal ablation island (Island \#3) in each HOA map. Each of the HOA ablation map islands were quantitatively characterized in terms of distance from center, position, orientation, sphericity, diameter, area, and depth.

keratometry (Kmax) and the depth of the peripheral crescents $(R=0.70 ; P=0.0132)$, between the corneal posterior radius of the curvature (PRC) and the ablation depth of the paracentral inferotemporal ablation island $(R=-0.74$; $P=0.0054)$, between the Pentacam Progression Index (PPI) and the ablation depth of the paracentral inferotemporal ablation island $(R=0.58 ; P=0.0485)$, and between the Belin/Ambrosio enhanced ectasia total deviation (BAD-D) and the ablation depth of all ablation islands $(R=0.71 ; P=0.0101)$. Other moderate to strong statistically significant correlations are reported in Table 3.

\section{Discussion}

The WaveLight Contoura planning software combines anterior corneal HOA Zernike coefficients (C6 to C27) from high-resolution Placido disc topographer scans (WaveLight Topolyzer VARIO) to generate an eyespecific anterior corneal HOA ablation map that is used to differentially remove small amounts of corneal tissue within the spherocylindrical ablation. This corneal HOA ablation map graphically represents the shapes, locations and depths of the excimer laser tissue removal patterns that are delivered onto the cornea to correct the anterior 


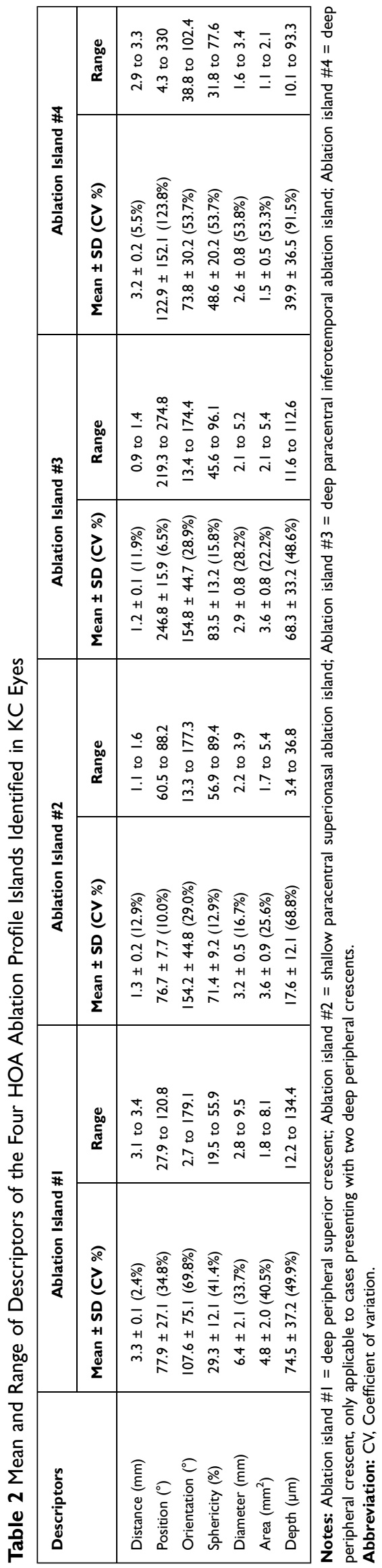

corneal surface HOA wavefront errors. The present pilot and proof-of-concept study is the first to analyze this map in order to quantify the graphical representation of corneal HOAs to be treated in KC eyes. Seven quantitative parameters from 4 ablation islands were derived. Unlike Zernike HOA values, these maps provide additional information related to position, eccentricity, depth, orientation and shape of anterior corneal HOAs to be treated. These new metrics can be used to describe features seen in $\mathrm{KC}$ eyes.

The current study identified four consistently recurring ablation islands on HOA ablation map imaging in $\mathrm{KC}$ eyes. These consisted of 2 elliptical paracentral mirror image ablation islands of equal sphericity and area, with a deeper inferotemporal ellipse (92\% of eyes inferotemporal) and its shallow corresponding superonasal ellipse (100\% of eyes superonasal). Both paracentral ablation islands had highly reproducible angular positions and distances from center, always within a $3.2 \mathrm{~mm}$ diameter central ring (1.6 mm radius from center). All eyes also displayed one or two superior, more peripheral crescents of various lengths consistently within the $6-7 \mathrm{~mm}$ diameter central ring, positioned in the superior quadrant in $94 \%$ of cases, and nasally in $70 \%$ of cases. The orientation of the superior crescents was nearly perpendicular to the position of the paracentral ellipses. The relationship between the paracentral ellipses and the superior peripheral crescents above them was also consistent, with similar average positions $\left(76.7^{\circ}\right.$ vs $\left.77.9^{\circ}\right)$, and with strongly correlated angular positions $(R=0.68)$ and depths $(R=0.94)$.

The inferotemporal deeper ellipse was visually coincident with the corneal thinnest spot, and with the peaks of both the anterior and posterior elevation topography/tomography maps. A comparison to topography elevation maps revealed that on average, the inferotemporal deeper island was identical in distance to center $(1.2 \pm 0.2 \mathrm{~mm}$ vs. $1.2 \pm$ $0.2 \mathrm{~mm} ; P=0.8486$ ), to the cone apex on elevation maps, with a maximum difference of $0.3 \mathrm{~mm}$ between the two. Similarly, the inferotemporal angular position of the deep paracentral ablation island $\left(246.8 \pm 15.9^{\circ}\right)$ was consistent with previously reported cone apex position, most commonly between $240^{\circ}$ and $270^{\circ} .{ }^{25}$ These findings support the consistently identifiable inferotemporal "hot spot" as representing the apical cone.

This novel, highly recognizable pattern seen in $\mathrm{KC}$ eyes is clinically relevant to the practicing surgeon, who as part of their workflow, could use the normalized colorcoded HOA ablation map as an indicator that $\mathrm{KC}$ may be 


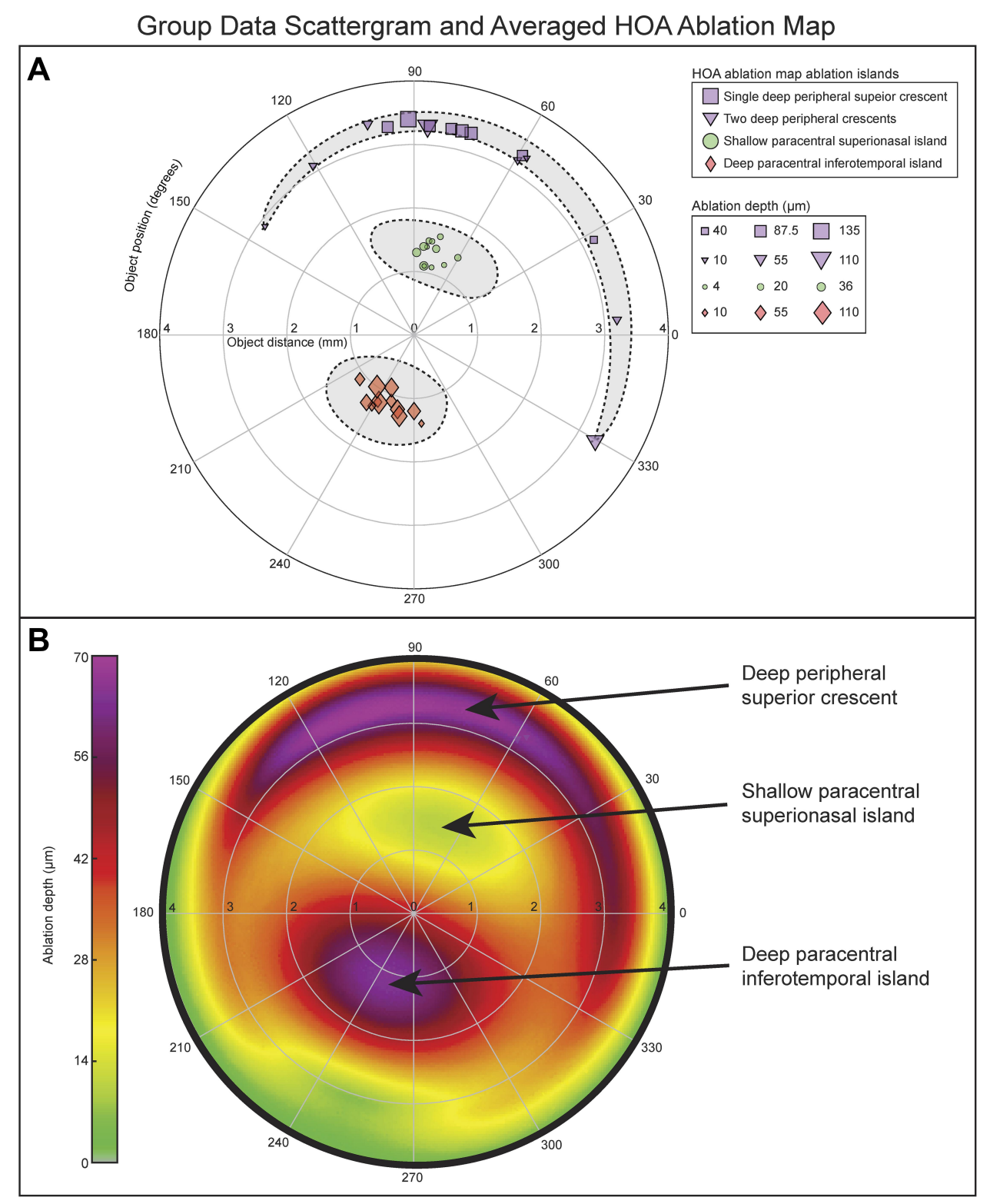

Figure 4 (A) Group data of the four ablation islands. The yellow/green superonasal ablation islands (island \#2; green circles) and the red/purple inferotemporal ablation islands (Island \#3; red diamonds) were highly reproducible in terms of distance from center and angular position, while their ablation depth varied more. The distance from center of the deep peripheral ablation islands (Island \#I and Island \#4; purple markers) was highly reproducible, with a larger range of depth. (B) Composite HOA ablation map obtained by averaging the HOA ablation map of all $12 \mathrm{KC}$ cases.

present. Identifying this pattern should lead to further review to confirm the diagnosis, in the same way a quick glance at inferior keratometric steepening triggers additional investigation. Future studies are needed to determine if other clinical conditions create a similar pattern, and if so, should quantify differences and establish discriminating values.

The characteristic arrangement of the paracentral deep and shallow ablation islands, in direct opposition to each other, seen in $100 \%$ of the KC HOA ablation maps, explains the differential corneal ablation required to correct a localized corneal elevation ( $\mathrm{KC}$ cone protrusion) causing comatic aberration. The peripheral crescent ablation also contributes to the comatic correction by effectuating a relative elevation superocentrally, further levelling out the cone. This explains why very strong correlations were found between the paracentral islands and the peripheral crescent in both depth $(R=0.94)$ and position $(R=$ $0.68)$. While the presence of concomitant spherical aberration and trefoil affect the position, shape and depth of the various ablation islands reported, coma was always the dominant aberration. 
Table 3 Pearson Correlation Between Ablation Depth and Pentacam Indices

\begin{tabular}{|l|l|l|l|}
\hline Parameters & $\begin{array}{l}\text { Depth of Island } \\
\mathbf{\# I}\end{array}$ & $\begin{array}{l}\text { Depth of Island } \\
\mathbf{\# 2}\end{array}$ & $\begin{array}{l}\text { Depth of Island } \\
\mathbf{\# 3}\end{array}$ \\
\hline Kmax & $0.70(0.0132)$ & $0.03(0.9383)$ & $0.64(0.0254)$ \\
ARC & $-0.49(0.1057)$ & $0.03(0.9417)$ & $-0.40(0.1945)$ \\
PRC & $-0.66(0.0204)$ & $-0.55(0.0645)$ & $-0.74(0.0054)$ \\
TP & $-0.52(0.0824)$ & $-0.38(0.2166)$ & $-0.35(0.2646)$ \\
ARTmax & $-0.33(0.2938)$ & $-0.28(0.3729)$ & $-0.35(0.2646)$ \\
PPI & $0.44(0.1574)$ & $0.55(0.0619)$ & $0.58(0.0485)$ \\
BAD-D & $0.69(0.0132)$ & $0.58(0.0459)$ & $0.71(0.0101)$ \\
ACA & $0.56(0.0572)$ & $0.11(0.7336)$ & $0.46(0.1333)$ \\
\hline
\end{tabular}

Notes: Values reported as Pearson correlation coefficient R. P-values are reported in brackets. Bold values highlight statistically significant correlations. Island \#I = deep peripheral superior crescent; Island \#2 = shallow paracentral superonasal ablation island; Island \#3 = deep paracentral inferotemporal ablation island. Abbreviations: $\mathrm{Kmax}$, maximal keratometry; ARC, anterior radius of curvature; PRC, posterior radius of curvature; TP, thinnest point; ARTmax, maximum Ambrosio relational thickness; PPI, Pentacam progression index; BAD-D, Belin/ Ambrosio enhanced ectasia total deviation; ACA, anterior corneal astigmatism.

The characteristic high coma ablation pattern was consistently identifiable in all eyes regardless of the position of the decentered cone. This pattern is comparable to the vertical coma-dominant arrangement recognizable on Topolyzer, Pentacam, Wavescan, and other wavefront analyzer wavefront maps in $\mathrm{KC} .{ }^{26-30}$ Since any decentered cone will create coma-dominant optics, this comatic pattern should also be present in atypical $\mathrm{KC}$ eyes, where the cone is not inferotemporal, but is nasal, central, or superior. Higher-order Zernike coefficients are used to construct an HOA wavefront map, producing a graphical and color-coded image of the ocular or corneal wavefront that produces this pattern. The wavefront phase is advanced superiorly because of the inferior shift of the cone's apex, leading to the familiar superior red and inferior blue opposing ellipses. The pattern that originates from corneal wavefront maps though are limited, in that no information is provided for the specific corneal location of the irregularity that produced the aberration. The HOA ablation map is unique in that it provides a quantitative measure (ablation depth) of aberrations while also localizing them on the corneal surface.

Anterior surface corneal HOA Zernike coefficients have been studied in diagnosing $\mathrm{KC}$, with coma-like aberrations thought to be good indicators for early detection and grading of keratoconus. ${ }^{26,30-33}$ However, conflicting results have not yielded adequate sensitivity and specificity to rely on this metric. Since the HOA ablation map provides additional graphical and depth data regarding HOAs, it may improve diagnostic ability beyond just Zernike coefficients alone.
The maximal depth in the HOA ablation map was previously found to be directly and highly correlated to the amount of anterior corneal HOAs, with total coma having the highest correlation. ${ }^{34}$ Our previous study showed that the maximal depth in normal eyes $(6.5 \mathrm{~mm}$ optical zone) was $8.02 \pm 3.00 \mu \mathrm{m}$, compared to $74.5 \pm 37.2$ $\mu \mathrm{m}$ in the current study's $\mathrm{KC}$ eyes, which is 9.3-fold higher than normals. ${ }^{14}$ In addition, the ablation depth was found to have a larger range of values and a higher coefficient of variation for all ablation islands, which may reflect the varying severity of $\mathrm{KC}$. Pearson correlations between the maximum ablation depth of the paracentral inferotemporal ablation island and Pentacam indices used for keratoconus grading revealed Kmax, PRC, PPI and BAD-D having strong correlations. Considering that the described paracentral inferotemporal ablation island is coincident with the apical cone, the ablation depth metric of this island may lend itself to be a new descriptor of $\mathrm{KC}$ severity. Further study to determine an ablation depth threshold for normals, subclinical and clinical $\mathrm{KC}$ could prove useful.

Delgado (2016) previously reported that coma aberrations were significantly correlated with keratoconus severity $(\mathrm{R}=0.60){ }^{35}$ The current study found higher correlations (up to $23 \%$ higher) between the inferotemporal ablation island's maximum depth and Pentacam grading indices (Kmax, PPI, PRC, and BAD-D), suggesting that the HOA ablation map may have the potential to increase the sensitivity and specificity of $\mathrm{KC}$ diagnosis and grading compared to individual corneal Zernike coefficients alone. Of note are cases \#7 and \#8, the right and left eye of the same patient. The least affected eye (OS, case \#8) showed no signs of $\mathrm{KC}$ on corneal epithelium mapping, while the HOA ablation map revealed $\mathrm{KC}$-like features, supporting the potential discriminating ability of the map.

Limitations of this study include its small sample size, as is typical for a proof-of-concept investigation. Future larger studies are needed to evaluate these novel HOA ablation map descriptors and HOA depth measurements. Comparison to existing technologies for early keratoconus detection in subclinical or form-fruste keratoconus as well as the role in determining $\mathrm{KC}$ disease progression warrants further investigation.

In summary, this pilot study qualitatively and quantitively characterizes the features of the HOA ablation map in $\mathrm{KC}$ eyes and adds to objective characteristics of $\mathrm{KC}$ corneal irregularities evidenced by other imaging 
modalities. $\mathrm{KC}$ eyes, with an inferotemporal cone, show a distinct easily recognizable pattern on HOA ablation maps. An arrangement of two ablation ellipses is seen, one deeper inferotemporal and a shallower superonasal, in direct mirror-like opposition to each other within the $3.2 \mathrm{~mm}$ diameter central ring. These are accompanied by 1 or 2 longer crescents of ablation above, confined within the 6-7 $\mathrm{mm}$ ring, whose mostly superonasal position correlated to the central ellipses. The deep inferotemporal ellipse "hot spot" is coincident with the topographical apical cone and its ablation depth strongly correlates to Pentacam BAD-D KC indices. HOA map ablation depth, as well as the other ablation island features, should be further studied to develop cut-off values with their corresponding sensitivity and specificity for differentiation of normals, subclinical and clinical keratoconus. Novel information can be extracted from the corneal HOA ablation map giving it the potential to be a new modality to diagnose, and grade $\mathrm{KC}$. The concepts presented in this pilot study would be applicable to any topography-guided platform.

\section{Funding}

There is no funding to report.

\section{Disclosure}

Dr Wallerstein and Cohen have indirect ownership in LASIK MD clinics. The authors have no conflict to disclose and no financial interest in the subject matter or materials presented in the current paper.

\section{References}

1. Garcia-Ferrer FJ, Akpek EK, Amescua G, et al. American Academy of Ophthalmology preferred practice pattern $\mathrm{C}$, external disease P. corneal ectasia preferred practice pattern(R). Ophthalmology. 2019;126:P170-P215. doi:10.1016/j.ophtha.2018.10.021

2. Kennedy RH, Bourne WM, Dyer JA. A 48-year clinical and epidemiologic study of keratoconus. Am J Ophthalmol. 1986;101:267-273. doi:10.1016/0002-9394(86)90817-2

3. Gorskova EN, Sevost'ianov EN. Epidemiology of keratoconus in the Urals. Vestn Oftalmol. 1998;114:38-40.

4. Jonas JB, Nangia V, Matin A, Kulkarni M, Bhojwani K. Prevalence and associations of keratoconus in rural Maharashtra in central India: the Central India Eye and Medical Study. Am $J$ Ophthalmol. 2009;148:760-765. doi:10.1016/j.ajo.2009.06.024

5. Martinez-Abad A, Pinero DP. New perspectives on the detection and progression of keratoconus. $J$ Cataract Refract Surg. 2017;43:1213-1227. doi:10.1016/j.jcrs.2017.07.021

6. Zhang X, Munir SZ, Sami Karim SA, Munir WM. A review of imaging modalities for detecting early keratoconus. Eye. 2020:1-15.

7. Beckman KA, Gupta PK, Farid M, Committee ACC., et al. Corneal crosslinking: current protocols and clinical approach. J Cataract Refract Surg. 2019;45:1670-1679. doi:10.1016/j.jcrs.2019.06.027
8. Randleman JB, Russell B, Ward MA, Thompson KP, Stulting RD. Risk factors and prognosis for corneal ectasia after LASIK. Ophthalmology. 2003;110:267-275. doi:10.1016/S0161-6420(02) 01727-X

9. Randleman JB, Trattler WB, Stulting RD. Validation of the ectasia risk score system for preoperative laser in situ keratomileusis screening. Am J Ophthalmol. 2008;145:813-818. doi:10.1016/j. ajo.2007.12.033

10. Randleman JB, Woodward M, Lynn MJ, Stulting RD. Risk assessment for ectasia after corneal refractive surgery. Ophthalmology. 2008;115(1):37-50. doi:10.1016/j.ophtha.2007.03.073

11. Pinero DP, Alio JL, Aleson A, Escaf Vergara M, Miranda M. Corneal volume, pachymetry, and correlation of anterior and posterior corneal shape in subclinical and different stages of clinical keratoconus. $J$ Cataract Refract Surg. 2010;36(5):814-825. doi:10.1016/j. jcrs.2009.11.012

12. Vega-Estrada A, Mimouni M, Espla E, Alio Del Barrio J, Alio JL. Corneal epithelial thickness intrasubject repeatability and its relation with visual limitation in keratoconus. $A m \quad J$ Ophthalmol. 2019;200:255-262. doi:10.1016/j.ajo.2019.01.015

13. Saad A, Gatinel D. Evaluation of total and corneal wavefront high order aberrations for the detection of forme fruste keratoconus. Invest Ophthalmol Vis Sci. 2012;53:2978-2992. doi:10.1167/iovs.11-8803

14. Wallerstein A, Gauvin M, Cohen M. Effect of anterior corneal higher-order aberration ablation depth on primary topography-guided LASIK outcomes. J Refract Surg. 2019;35 (12):754-762. doi:10.3928/1081597X-20191021-02

15. Faria-Correia F, Ribeiro S, Monteiro T, Lopes BT, Salomao MQ, Ambrosio R Jr. Topography-guided custom photorefractive keratectomy for myopia in primary eyes with the wavelight EX500 platform. J Refract Surg. 2018;34:541-546. doi:10.3928/ 1081597X-20180705-03

16. Stulting DR, Fant BS, Group TCS, et al. Results of topography-guided laser in situ keratomileusis custom ablation treatment with a refractive excimer laser. $J$ Cataract Refract Surg. 2016;42(1):11-18. doi:10.1016/j.jcrs.2015.08.016

17. Wallerstein A, Gauvin M, Qi SR, Bashour M, Cohen M. Primary topography-guided LASIK: treating manifest refractive astigmatism versus topography-measured anterior corneal astigmatism. $J$ Refract Surg. 2019;35:15-23. doi:10.3928/1081597X-20181113-01

18. Motwani M. A protocol for topographic-guided corneal repair utilizing the US Food and Drug Administration-approved wavelight contoura. Clin Ophthalmol. 2017;11:573-581. doi:10.2147/OPTH. S127855

19. Amsler M. Keratocone classique et keratocone fruste; arguments unitaires. Ophthalmologica. 1946;111:96-101. doi:10.1159/ 000300309

20. Kojima T, Nishida T, Nakamura T, et al. Keratoconus screening using values derived from auto-keratometer measurements: a multicenter study. Am $J$ Ophthalmol. 2020;215:127-134. doi:10.1016/j. ajo.2020.02.017

21. Baenninger PB, Bodmer NS, Bachmann LM, et al. Keratoconus characteristics used in randomized trials of surgical interventions-a systematic review. Cornea. 2020;39:615-620. doi:10.1097/ICO.0000000000002202

22. Belin MW, Duncan JK. Keratoconus: the ABCD grading system. Klin Monbl Augenheilkd. 2016;233(06):701-707. doi:10.1055/ s-0042-100626

23. Wallerstein A, Caron-Cantin M, Gauvin M, Adiguzel E, Cohen M. Primary topography-guided LASIK: refractive, visual, and subjective quality of vision outcomes for astigmatism 2.00 diopters. $J$ Refract Surg. 2019;35:78-86. doi:10.3928/1081597X-20181210-01

24. Wallerstein A, Gauvin M, Adiguzel E, et al. Clinically significant laser in situ keratomileusis flap striae. J Cataract Refract Surg. 2017;43:1523-1533. doi:10.1016/j.jcrs.2017.09.023 
25. McMahon TT, Anderson RJ, Szczotka-Flynn LB, et al., Group CS. Gender differences in cone apex location in keratoconus. Invest Ophthalmol Vis Sci. 2004;45:2894.

26. Kosaki R, Maeda N, Bessho K, et al. Magnitude and orientation of zernike terms in patients with keratoconus. Invest Ophthalmol Vis Sci. 2007;48:3062-3068. doi:10.1167/iovs.06-1285

27. Atchison DA, Mathur A, Read SA, et al. Peripheral ocular aberrations in mild and moderate keratoconus. Invest Ophthalmol Vis Sci. 2010;51:6850-6857. doi:10.1167/iovs.10-5188

28. Nakagawa T, Maeda N, Kosaki R, et al. Higher-order aberrations due to the posterior corneal surface in patients with keratoconus. Invest Ophthalmol Vis Sci. 2009;50:2660-2665. doi:10.1167/iovs.08-2754

29. Pantanelli S, MacRae S, Jeong TM, Yoon G. Characterizing the wave aberration in eyes with keratoconus or penetrating keratoplasty using a high-dynamic range wavefront sensor. Ophthalmology. 2007;114:2013-2021. doi:10.1016/j.ophtha.2007.01.008

30. Nilforoushan MR, Speaker M, Marmor M, et al. Comparative evaluation of refractive surgery candidates with placido topography, orbscan II, pentacam, and wavefront analysis. J Cataract Refract Surg. 2008;34:623-631. doi:10.1016/j.jcrs.2007.11.054
31. Barbero S, Marcos S, Merayo-Lloves J, Moreno-Barriuso E. Validation of the estimation of corneal aberrations from videokeratography in keratoconus. J Refract Surg. 2002;18:263-270.

32. Buhren J, Kuhne C, Kohnen T. Defining subclinical keratoconus using corneal first-surface higher-order aberrations. Am J Ophthalmol. 2007;143:381-389. doi:10.1016/j.ajo.2006.11.062

33. Saad A, Gatinel D. Combining placido and corneal wavefront data for the detection of forme fruste keratoconus. J Refract Surg. 2016;32:510-516. doi:10.3928/1081597X-20160523-01

34. Wallerstein A, Gauvin M, Cohen M. The relationship between preoperative anterior corneal higher-order aberrations and topography-guided excimer ablation depth. J Refract Surg. 2020. doi:10.3928/1081597X-20200611-02

35. Delgado S, Velazco J, Delgado Pelayo RM, Ruiz-Quintero N. Correlation of higher order aberrations in the anterior corneal surface and degree of keratoconus measured with a scheimpflug camera. Arch Soc Esp Oftalmol. 2016;91:316-319. doi:10.1016/j. oftal.2016.01.014
Clinical Ophthalmology

\section{Publish your work in this journal}

Clinical Ophthalmology is an international, peer-reviewed journal covering all subspecialties within ophthalmology. Key topics include: Optometry; Visual science; Pharmacology and drug therapy in eye diseases; Basic Sciences; Primary and Secondary eye care; Patient Safety and Quality of Care Improvements. This journal is indexed on PubMed
Dovepress

Central and CAS, and is the official journal of The Society of Clinical Ophthalmology (SCO). The manuscript management system is completely online and includes a very quick and fair peer-review system, which is all easy to use. Visit http://www.dovepress.com/ testimonials.php to read real quotes from published authors. 